\title{
Oxidative stress measurement by in vivo electron spin resonance spectroscopy in rats with streptozotocin-induced diabetes
}

\author{
T. Sano ${ }^{1}$, F. Umeda ${ }^{1}$, T. Hashimoto ${ }^{1}$, H. Nawata ${ }^{1}$, H. Utsumi ${ }^{2}$ \\ ${ }^{1}$ Third Department of Internal Medicine, Faculty of Medicine, Kyushu University, Fukuoka, Japan \\ ${ }^{2}$ Department of Biophysics, Faculty of Pharmaceutical Sciences, Kyushu University, Fukuoka, Japan
}

\begin{abstract}
Summary Enhanced oxidative stress in diabetic patients may contribute to the pathogenesis of diabetic angiopathy. We have recently developed a method to determine the electron spin resonance (ESR, electron paramagnetic resonance; EPR) of reactive oxygen species and free radicals in vivo, using the nitroxide derivative, carbamoyl-PROXYL as a probe. In this study, diabetes was induced in Wistar rats by streptozotocin (STZ) injection $(65 \mathrm{mg} / \mathrm{kg}$, body weight, intravenously). Two, 4 , and 8 weeks later, the animals received carbamoyl-PROXYL $(300 \mathrm{nmol} / \mathrm{g}$, intravenously), and ESR was measured at the upper abdominal level at a frequency of $300 \mathrm{MHz}$. The intensity of the carbamoyl-PROXYL ESR signal decreased gradually after the injection, and the spin clearance rate was determined over the first $5 \mathrm{~min}$. At all time points, the spin clearance rate was significantly greater in the diabetic rats than in control rats. Moreover, the spin clearance rate in the diabetic rats was significantly correlated with urinary malondialdehyde (MDA) levels, which serve as a marker
\end{abstract}

for lipid peroxidation. Daily treatment with 4 units neutral protamin Hagedorn (NPH) insulin for 4 weeks reduced the spin clearance rate in the diabetic rats. Simultaneous injection of carbamoyl-PROXYL and superoxide dismutase reduced the spin clearance rate in the diabetic rats in a dose-dependent manner. Injection of the antioxidant $\alpha$-tocopherol (40 mg/kg, intraperitoneally) for 2 weeks restored the spin clearance rate in the diabetic rats without concomitant glycaemic restoration. These results suggest that a diabetic state enhances the generation of free radicals in vivo, and that both glycaemic control and antioxidant treatment can reduce this oxidative stress. Non-invasive in vivo ESR measurement may be useful for evaluating oxidative stress in diabetes. [Diabetologia (1998) 41: 1355-1360]

Keywords Oxidative stress, nitroxide radical, electron spin resonance, streptozotocin, superoxide dismutase, $\alpha$-tocopherol.
The generation of reactive oxygen species and free radicals has been shown to be increased in patients with diabetes mellitus [1,2]. Free radicals generated through glycation may accelerate oxidation reactions in the vascular walls, thereby contributing significant-

Received: 21 January 1998 and in final revised form: 12 June 1998

Corresponding author: Dr. T. Sano, Maidashi 3-1-1, Higashiku, Fukuoka 812, Japan

Abbreviations: ESR, Electron spin resonance; STZ, streptozotocin; MDA, malondialdehyde; SOD, superoxide dismutase; TBA, thiobarbituric acid. ly to the pathogenesis of diabetic angiopathy [3-5]. Because free radicals are highly unstable and reactive, and difficult to measure in vivo, most previous experiments have been performed in vitro or ex vivo. The formation of free radicals may be regulated in a complicated manner, requiring new methods for direct determination.

Free radicals have unpaired electrons, which can be detected selectively and sensitively by electron spin resonance (ESR) spectroscopy. Conventional $\mathrm{X}$-band ESR spectroscopy, however, cannot be used for water-rich samples, such as living animals. Recently, a low-frequency ESR apparatus has been designed that allows in vivo measurement of free radi- 
cals in living animals [6]. On the other hand, nitroxide radicals have been used to examine the changes in membrane fluidity and protein conformation. Utsumi et al. [7] have proposed that they could be also used as spin probes for in vivo free radical reactions. These reactions result in a decrease in ESR signals $[8,9]$. For example, Utsumi [10] demonstrated that a nitroxide radical could be used as a spin probe for in vivo ESR measurement of free radicals in living mice.

It was reported that dietary supplementation of the antioxidant $\alpha$-tocopherol (vitamin E) may be associated with a significantly reduced risk of coronary heart disease in men and middle-aged women [11, 12]. This may result from $\alpha$-tocopherol's effect on prostaglandin and cytokine production, as well as from its inhibition of the oxidative modification of low-density lipoproteins [13].

In the present study, we determined oxidative stress in rats with streptozotocin (STZ) induced diabetes, using the newly developed in vivo ESR method. In addition, we evaluated the effect of $\alpha$-tocopherol on free radical formation in these diabetic rats.

\section{Materials and methods}

Animals. Female Wistar rats were bred under pathogen-free conditions at the Kyushu University Animal Center, Fukuoka, Japan. The animals had free access to tap water and standard chow (Clea Japan Inc., Tokyo, Japan) containing 50.1 \% carbohydrate, $25.1 \%$ protein, $7.1 \%$ minerals, $4.5 \%$ fat, and $4.3 \%$ cellulose until the start of the experiment.

At 6 weeks of age, the rats were injected in the tail vein with $65 \mathrm{mg} / \mathrm{kg}$ body weight STZ (Sigma, St. Louis, Mo., USA) in $0.9 \% \mathrm{NaCl}$ after overnight fasting. One or two days after the injection, the development of diabetes was verified by the presence of hyperglycaemia, polyuria and glycosuria. The rats were used for the following experiments 2,4 , or 8 weeks after the onset of diabetes.

ESR measurement. Rats were anaesthetized by intraperitoneal injection of pentobarbital $(50 \mathrm{mg} / \mathrm{kg})$ and fixed on a handmade teflon holder. Sterilized 3-carbamoyl-2,2,5,5-tetramethylpyrrolidin-1-yloxy (carbamoyl-PROXYL; Aldrich Chemical Co. Milw., WI, USA) solution (300 mmol/l) was injected into the rats' tail vein at a concentration of $1 \mu \mathrm{l} / \mathrm{g}$ body weight. Immediately after the injection, the ESR spectrum was measured at the upper abdominal level, using an ESR spectrometer equipped with a $300 \mathrm{MHz}$ microwave power unit and a large size (70 mm diameter) loop-gap resonator which was newly constructed by JEOL (Akishima, Japan) in collaboration with $\mathrm{H}$. Utsumi. The microwave power was set at $3.0 \mathrm{~mW}$. The amplitude of the $100 \mathrm{kHz}$ field modulation was $0.1 \mathrm{mT}$. The external magnetic field was swept from 5 to $15 \mathrm{mT}$ at scan rate of $5 \mathrm{mT} /$ min. The measurement of 2,2-diphenyl-1-(2,4,6-trinitrophenyl)hydrazyl (DPPH) powder as a standard revealed that the sensitivity of the $300 \mathrm{MHz}$ ESR spectrometer is comparable to that of an l-band spectrometer.

Malondialdehyde (MDA) measurement. The levels of MDA in 24-h urine samples were measured fluorometrically using thiobarbituric acid (TBA) [14]. The concentration of the
TBA reaction products was determined using excitation at $515 \mathrm{~nm}$ and emission at $553 \mathrm{~nm}$. The resulting MDA values were corrected for creatinine levels in the urine.

$\alpha$-tocopherol measurement. The serum levels of $\alpha$-tocopherol in rats were measured by HPLC as described by Abe and Katsui [15].

Experimental protocols. Experiment 1: ESR measurements were performed in diabetic rats at 2,4 , and 8 weeks after the onset of diabetes and compared with the values from agematched control animals. In addition, five animals from each group (except those at 2 weeks after the onset of diabetes) were randomly chosen for MDA analysis. These rats were transferred to stainless-steel metabolic cages, and their urine was collected for $24 \mathrm{~h}$. The urine samples were stored at $-20^{\circ} \mathrm{C}$ until further analysis. Experiment 2: Diabetic rats at 2 weeks after the onset of diabetes were injected simultaneously with carbamoyl-PROXYL and $0,100,500$, or 1,000 units superoxide dismutase (SOD) (Wako Pure Chemical Industries, Osaka, Japan), followed immediately by in vivo ESR measurement. Experiment 3: Diabetic rats were treated by subcutaneous injection of 4 units neutral protamin Hagedorn (NPH) insulin (Eli Lilly \& Co., Ind., USA) every evening starting immediately after the onset of diabetes. After 4 weeks, in vivo ESR measurements were performed and compared with untreated diabetic rats and control rats. Experiment 4: Diabetic rats and control rats were injected intraperitoneally with $40 \mathrm{mg} / \mathrm{kg} \alpha$-tocopherol or placebo vehicle (HCO; polyethylene 60-hydrogenated castor oil) (kindly provided by Eisai Co., Ltd., Tokyo, Japan) every morning, beginning at the onset of diabetes. After 2 weeks, in vivo ESR measurements were performed in all animals.

All experimental protocols were reviewed by the ethics committee for animal experiments at the Faculty of Medicine, Kyushu University and carried out according to the Guidelines for Animal Experiments of the Faculty of Medicine, Kyushu University, as well as Law No. 105 and Notification No. 6 of the Japanese Government.

Statistical analysis. All data are expressed as means \pm SEM unless indicated otherwise. Student's $t$-test was used to determine the significance of differences between two groups, and Fisher's PLSD or Sheffe's multiple comparison test was used to compare differences among more than three groups. Pearson's coefficient test was used for determining correlation between two groups. $P$ values of less than 0.05 were accepted as statistically significant.

\section{Results}

To determine the levels of reactive oxygen species and free radicals by in vivo ESR measurements, we used carbamoyl-PROXYL as a spin probe. Immediately after its administration, the probe began to be converted into its hydroxylamine, which produces no ESR signal (Fig. 1 a). The typical ESR spectra of nitroxide radical was detected at the rats' upper abdominal level (Fig. 1b). As a result, the intensity of the ESR signal decreased gradually, and no line broading due to immobilization of the probe was observed within $5 \mathrm{~min}$. A semilogarithmic plot of the time course of the ESR signals yielded a signal decay curve 
a Carbamoyl-PROXYL

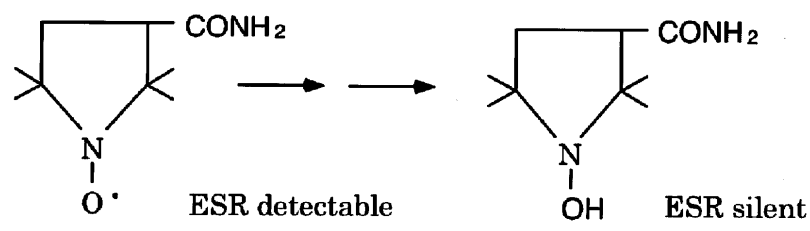

b

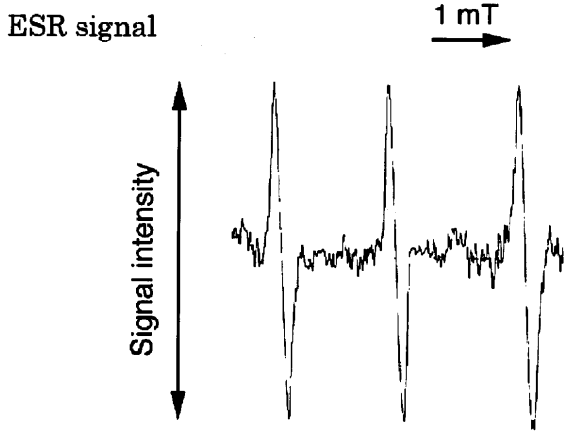

c Clearance curve of carbamoyl-PROXYL

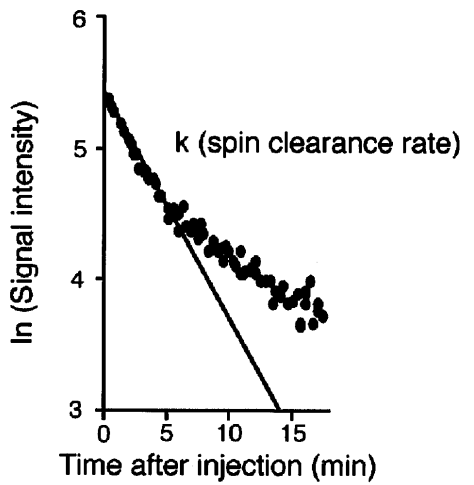

Fig. 1. (a) The nitroxide radical, carbamoyl-PROXYL, in vivo reacts with biological free radicals, such as reactive oxygen species. As a result, carbamoyl-PROXYL is reduced to the corresponding hydroxylamine, leading to the loss of paramagnetism. (b) The ESR spectrum of carbamoyl-PROXYL exhibits a typical triplet signal. The ESR signal intensity decreases gradually after intravenous administration. (c) The signal decay curve of carbamoyl-PROXYL was obtained by semilogarithmically plotting the peak heights of the ESR signals. The initial kinetic constant ( $\mathrm{k}$; spin clearance rate) was calculated from the slope of the signal decay curve

for carbamoyl-PROXYL. The curve was almost linear over the first $5 \mathrm{~min}$ of decay, allowing the calculation of initial velocity as the clearance constant (spin clearance rate) (Fig. 1 c). The spin clearance rate represents the rate of in vivo radical formation.

Comparison of the spin clearance rates in rats with STZ-induced diabetes and control animals demonstrated that the rates in the diabetic rats were significantly greater than those of the control animals at 2 weeks $(0.212 \pm 0.013, n=10 \quad$ vs $0.167 \pm 0.018$, $n=12 ; p<0.01), 4$ weeks $(0.228 \pm 0.019, n=12$ vs $0.178 \pm 0.008, \quad n=13 ; \quad p<0.01), \quad$ and 8 weeks

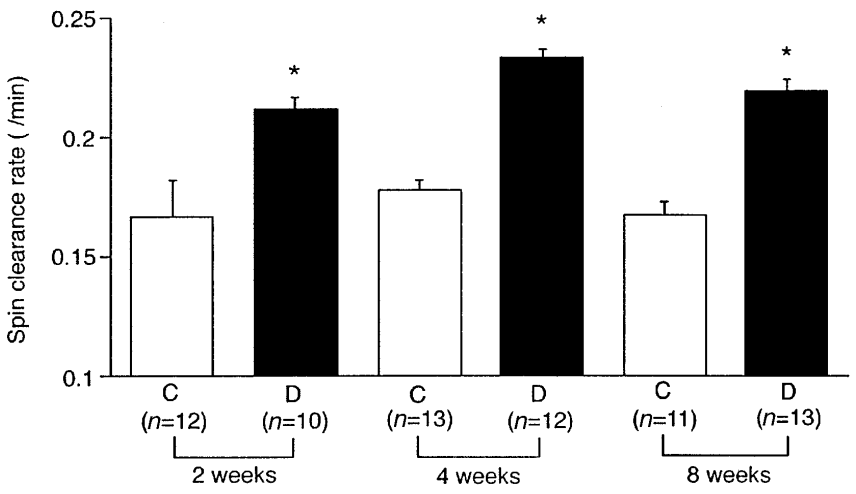

Fig. 2. Changes in the spin clearance rate in rats with STZ-induced diabetes (D) compared with nondiabetic control animals $(\mathrm{C})$. Each value represents the mean \pm SEM. $* p<0.01$ vs control

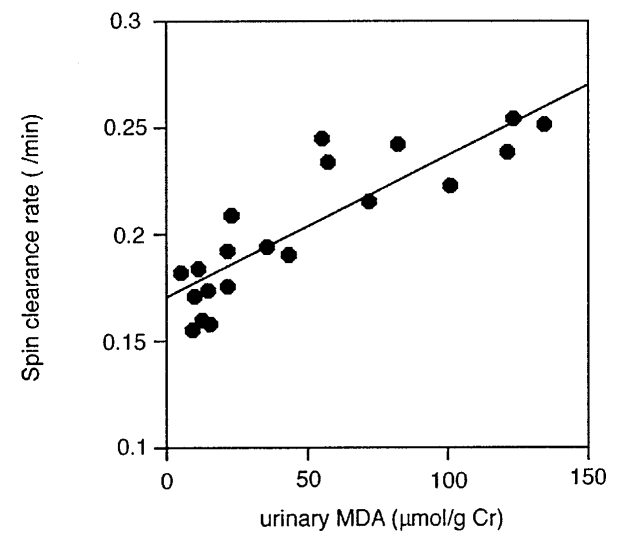

Fig. 3. Correlation between the spin clearance rate and urinary MDA value in rats at 4 and 8 weeks after the onset of diabetes, and in control animals $(r=0.87, p<0.0001, n=20)$

$(0.220 \pm 0.021, \quad n=13 \quad$ vs $\quad 0.167 \pm 0.019, \quad n=11$; $p<0.01$ ) of diabetes (Fig.2). Similarly, the MDA concentrations in 24-h urine samples from diabetic rats were significantly greater than those of the control rats after 4 weeks and 8 weeks $(80 \pm 12 \mu \mathrm{mol} /$ $\mathrm{gCr}, n=10$, vs $17 \pm 3, n=10 ; p<0.01)$. Moreover, a significant positive correlation existed between the urinary MDA levels and the spin clearance rate of diabetic rats and control rats at those time points $(r=0.87, p<0.01, n=20)$ (Fig. 3). The injection of SOD simultaneously with carbamoyl-PROXYL to diabetic rats 2 weeks after STZ injection reduced the increased spin clearance rates in those animals in a dose-dependent manner (1000 units: $0.174 \pm 0.008$, $n=5, \quad p<0.01 ; 500$ units: $0.179 \pm 0.018, \quad n=5$, $p<0.01 ; 100$ units: $0.188 \pm 0.020, n=5, p=0.07$ vs 0 unit: $0.212 \pm 0.013, n=10$ ) (Fig. 4). Conversely, the addition of SOD did not affect the spin clearance rate of the control animals. Inactivated SOD (corresponding to 1000 units) did not reduce the increased spin clearance rates in STZ-induced diabetes $(0.196 \pm 0.009)$. These results suggest that the in- 
Table 1. Effect of insulin treatment for 4 weeks following the onset of diabetes on the increased spin clearance rate of the diabetic rats

\begin{tabular}{lllll}
\hline & $n$ & Blood glucose $(\mathrm{mmol} / \mathrm{l})$ & $\mathrm{HbA}_{1 \mathrm{c}}(\%)$ & Spin clearance rate \\
\hline Control & 5 & $6.9 \pm 0.4$ & $2.4 \pm 0.1$ & $0.161 \pm 0.007$ \\
Diabetes & 5 & $22.8 \pm 1.7^{\mathrm{a}}$ & $8.6 \pm 0.3^{\mathrm{a}}$ & $0.229 \pm 0.006^{\mathrm{a}}$ \\
Diabetes + Insulin & 9 & $9.3 \pm 0.4^{\mathrm{a}, \mathrm{b}}$ & $3.6 \pm 0.2^{\mathrm{a}, \mathrm{b}}$ & $0.172 \pm 0.005^{\mathrm{b}}$ \\
\hline
\end{tabular}

Each value represents the mean \pm SEM. ${ }^{\mathrm{a}} p<0.01$ vs control. ${ }^{\mathrm{b}} p<0.01$ vs diabetes

Table 2. Effect of $\alpha$-tocopherol administration for 2 weeks following the onset of diabetes on the increased spin clearance rate in the diabetic rats

\begin{tabular}{lllccl}
\hline & & $n$ & Blood glucose $(\mathrm{mmol} / \mathrm{l})$ & Serum $\alpha$-tocopherol $(\mathrm{mg} / \mathrm{dl})$ & Spin clearance rate \\
\hline Control & placebo & 6 & $4.8 \pm 0.5$ & $6.0 \pm 0.5$ & $0.169 \pm 0.008$ \\
& $\alpha$-tocopherol & 6 & $5.2 \pm 0.3$ & $22.5 \pm 1.2^{\mathrm{a}}$ & $0.181 \pm 0.010$ \\
Diabetes & placebo & 6 & $16.2 \pm 2.3^{\mathrm{a}}$ & $2.7 \pm 0.6$ & $0.213 \pm 0.007^{\mathrm{a}}$ \\
& $\alpha$-tocopherol & 6 & $14.9 \pm 2.4^{\mathrm{a}}$ & $18.5 \pm 10.3^{\mathrm{b}}$ & $0.180 \pm 0.006^{\mathrm{b}}$ \\
\hline
\end{tabular}

Each value represents the mean \pm SEM. ${ }^{\mathrm{a}} p<0.01$ vs control + placebo. ${ }^{\mathrm{b}} p<0.01$ vs STZ + placebo

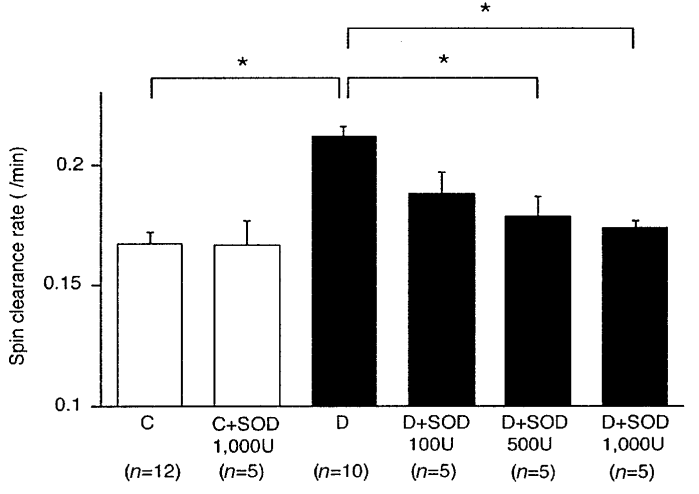

Fig. 4. Effect of SOD administration on the increased spin clearance rate in rats with STZ-induced diabetes at 2 weeks after the onset of diabetes. Each value represents the mean \pm SEM. $* p<0.01$. C; nondiabetic controls, D; untreated diabetic rats

creased spin clearance rates are due to the interaction with superoxide.

Daily insulin injections for 4 weeks following the onset of diabetes significantly improved the increased spin clearance rates of the diabetic rats and at the same time achieved glycaemic control and $\mathrm{HbA}_{1 \mathrm{c}}$ (Table 1). The administration of $\alpha$-tocopherol, in contrast, also restored the spin clearance rate in the diabetic rats, but did not alter their blood glucose levels. The spin clearance rates of the control rats were not significantly altered by $\alpha$-tocopherol administration (Table 2).

\section{Discussion}

In many reports the diabetic state has been shown to enhance oxidative stress; however, this has not been proved clearly or directly in vivo. In this study, we propose a new method for measuring free radicals in vivo in a diabetic model.

A semilogarithmic plot of the time course of the in vivo ESR signal peaks of carbamoyl-PROXYL showed a linear decay curve, indicating that the decay of carbamoyl-PROXYL follows first-order kinetics $[10,16,17]$. Nitroxide radicals such as carbamoylPROXYL are reduced to the corresponding hydroxylamine in vivo, resulting in the disappearance of their ESR signals [18]. In addition, a previous study found that superoxide anion radicals reduce nitroxides to hydroxylamines in the presence of thiols [8]. The addition of potassium ferricyanide, which oxidizes hydroxylamine to nitroxide, to collected blood has been shown to result in the reappearance of the ESR signals [19], indicating that nitroxide radicals lose their paramagnetism through a one-electron reduction. There should be other mechanisms which could account for the disappearance of the ESR signal distribution to the various tissues and excretion into urine over time. In this study, the initial velocity of nitroxide clearance was obtained within 5 min after the injection of the nitroxide radical. Moreover, we also confirmed that the decreased ESR signal was restored to the initial intensity within a short time by the addition of potassium ferricianide which oxidizes hydroxylamine to nitroxide in various tissue. This suggests that the changes in nitroxide clearance may be due mainly to one-electron reduction of carbamoyl-PROXYL to the corresponding hydroxylamine.

Conventional ESR spectrometers have significant limitations for analyses in living animals because the high-frequency microwaves used are absorbed by water, resulting in the development of high fever. To overcome this limitation, Froncisz and Hyde [20] and Berliner et al. [21] used ESR spectrometers with resonators to noninvasively measure radical formation in vivo. Furthermore, Utsumi et al. [6] studied is- 
chaemia-reperfusion injury in femoral muscle of mice by low-frequency in vivo ESR, using the nitroxide radical 4-amino-2,2,6,6-tetramethylpiperidine-1-yloxyl (amino-TEMPO) as a spin probe. The animals subjected to ischaemia-reperfusion showed a significantly greater spin clearance rate than control animals. Moreover, SOD restored the increased spin clearance rate to control levels. Higher levels of oxygen in the inhaled air also significantly increased the spin clearance rate measured at the upper abdominal level, a process that was reversed by several antioxidants [22]. These findings indicated that nitroxides are susceptible to oxidative stress and could be used as probes for in vivo ESR measurements.

In this study, we used in vivo ESR measurements to analyse oxidative stress in rats with experimentally induced diabetes. These analyses found that the spin clearance rate was increased in the diabetic rats, indicating that the oxidative stress was enhanced in these animals. Moreover, the animals' spin clearance rates were positively correlated with their urinary MDA levels, which indicate the extent of lipid peroxidation, thereby serving as an index of oxidative stress in vivo [23]. A previous study also demonstrated that urinary excretion of thiobarbituric acid-reactive substances was elevated in diabetic rats [24]. The addition of SOD ameliorated the increase in spin clearance rate, suggesting that the formation of free radicals and superoxide was increased in the diabetic rats. Several mechanisms have been proposed by which hyperglycaemia could increase the generation of free radicals [25-29]. Diabetes-inducing agents, such as alloxan and STZ, can themselves produce reactive oxygen species [30-33]. To exclude the potential influence of STZ, we also determined the spin clearance rate of insulin-treated rats with STZ-induced diabetes. Insulin treatment reduced the increased spin clearance rate in these animals, indicating that the increase was caused by the hyperglycaemia and not by the STZ. Moreover, $\alpha$-tocopherol treatment reduced the increased spin clearance rate of diabetic rats without achieving glycaemic control. These findings suggest that other mechanisms in addition to hyperglycaemia may enhance oxidative stress in the diabetic state.

In conclusion, the spin clearance rate in rats with STZ-induced diabetes was significantly greater than that in nondiabetic control animals. Simultaneous administration of SOD and carbamoyl-PROXYL restored the increased spin clearance rate of diabetic rats, as did treatment with insulin or $\alpha$-tocopherol. These results suggest that diabetes is associated with enhanced oxidative stress, due primarily to the hyperglycaemia-induced increase in the in vivo generation of free radicals, particularly superoxides. Furthermore, noninvasive in vivo ESR measurement may be useful for evaluating oxidative stress in diabetes.
We only used the carbamoyl-PROXYL as spin probe in this experiment. Some of the nitroxide radical also reacts in the same way. Therefore, if another nitroxide radical which distributes to the specific organ for example were used, this method would provide more useful information.

Acknowledgements. This work was supported by a Grant-inAid for Scientific Research (No. 01570646, No. 06557123, No. 07457530) from the Ministry of Education, Science and Culture, Japan.

\section{References}

1. Wolff SP, Jiang ZY, Hunt JV (1991) Protein glycation and oxidative stress in diabetes mellitus and aging. Free Radic Biol Med 10: 339-352

2. Oberley LW (1988) Free radicals and diabetes. Free Rad Biol Med 5: 113-124

3. Mullarkey CJ, Edelstein D, Brownlee M (1990) Free radical generation by early glycation products: a mechanism for accelerated atherogenesis in diabetes. Biochem Biophys Res Commun 173: 932-939

4. Baynes JW (1991) Role of oxidative stress in development of complications in diabetes. Diabetes 40: 405-412

5. Williamson JR, Chang K, Frangos M et al. (1993) Hyperglycemic pseudohypoxia and diabetic complications. Diabetes 42: 801-813

6. Swartz HM, Sentjurc M, Kocherginsky N (1995) Metabolism and distribution of nitroxides in vivo. In: Kocherginsky $\mathrm{N}$, Swartz HM (eds) Nitroxide spin labels reactions in biology and chemistry. CRC press, Inc., Boca Raton, Fla., pp 153-173

7. Utsumi H, Takeshita K, Miura Y, Masuda S, Hamada A (1993) In vivo EPR measurement of radical reaction in whole mice. Free Rad Res 19: 219-225

8. Finkelstein E, Rosen GM, Rauckman EJ (1984) Superoxide-dependent reduction of nitroxides by thiols. Biochim Biophys Acta 802: 90-98

9. Samuni A, Krishna CM, Riesz P, Finkelstein E, Russo A (1989) Superoxide reaction with nitroxide spin-adducts. Free Rad Biol Med 6: 141-148

10. Utsumi H (1995) In vivo ESR measurement of free radical reactions in living mice. Environ Mut Res Commun 16: 317-325

11. Rimm EB, Stampfer MJ, Ascherio A, Giovannucci E, Colditz GA, Willett WC (1993) Vitamin E consumption and the risk of coronary heart disease in men. N Engl J Med 328: 1450-1456

12. Stampfer MJ, Hennekens CH, Manson JE, Colditz GA, Rosner B, Willett WC (1993) Vitamin E consumption and the risk of coronary disease in women. N Engl J Med 328: 1444-1449

13. Martin A, Wu D, Baur W, Meydani SN, Blumberg JB, Meydani M (1996) Effect of vitamin E on human aortic endothelial cell responses to oxidative injury. Free Rad Biol Med 21: 505-511

14. Yagi K (1976) A simple fluorometric assay for lipoperoxide in blood plasma. Biochem Med 15: 212-216

15. Abe K, Katsui G (1975) Determination of tocopherols in serum by high speed liquid chromatography. Vitamins (Japan) 49: 259-263

16. Utsumi H, Masuda S, Muto E, Hamada A (1991) In vivo ESR studies on pharmacokinetics of nitroxide radicals in 
whole mice. In: Davies KJA (ed) Oxidative damage and repair. California, Pasedena, pp 165-170

17. Utsumi H, Muto E, Masuda S, Hamada A (1990) In vivo ESR measurement of free radicals in whole mice. Biochem Biophys Res Commun 172: 1342-1348

18. Takeshita K, Utsumi H, Hamada A (1993) Whole mouse measurement of paramagnetism-loss of nitroxide free radical in lung with a L-band ESR spectrometer. Biochem Mol Biol Int 29: 17-24

19. Gomi F, Utsumi H, Hamada A, Matsuo M (1993) Aging retards spin clearance from mouse brain and food restriction prevents its age-dependent retardation. Life Sciences 52: 2027-2033

20. Froncisz W, Hyde JS (1982) The loop-gap resonator: a new microwave lumped circuit ESR sample structure. J Magn Reson 47: 515-521

21. Berliner LJ, Fujii H, Wan X, Lukiewicz SJ (1987) Feasibility study of imaging a living murine tumor by electron paramagnetic resonance. Magn Reson Med 10: 266-272

22. Miura Y, Hamada A, Utsumi H (1995) In vivo ESR studies of antioxidant activity on free radical reaction in living mice under oxidative stress. Free Rad Res 22: 209-214

23. Draper HH, Polensek L, Hadley M, McGirr LG (1984) Urinary malondialdehyde as an indicator of lipid peroxidation in the diet and in the tissues. Lipids 19: 836-843

24. Gallaher DD, Csallany AS, Shoeman DW, Olson JM (1993) Diabetes increases excretion of urinary malonaldehyde conjugates in rats. Lipids 28: 663-666

25. Jiang ZY, Woollard AC, Wolff SP (1990) Hydrogen peroxide production during experimental protein glycation. FEBS Lett 268: 69-71
26. Kashiwagi A, Asahina T, Ikebuchi M et al. (1994) Abnormal glutathione metabolism and increased cytotoxicity caused by $\mathrm{H}_{2} \mathrm{O}_{2}$ in human umbilical vein endothelial cells cultured in high glucose medium. Diabetologia 37: 264-269

27. Barnett PA, Gonzalez RG, Chylack LT Jr, Cheng HM (1986) The effect of oxidation on sorbitol pathway kinetics. Diabetes 35: 426-432

28. Kawamura N, Ookawara T, Suzuki K, Konishi K, Mino M, Taniguchi N (1992) Increased glycated Cu,Zn-superoxide dismutase levels in erythrocytes of patients with insulin-dependent diabetes mellitus. J Clin Endocrinol Metab 74: 1352-1354

29. Paolisso G, D'Amore A, Volpe C et al. (1994) Evidence for a relationship between oxidative stress and insulin action in non-insulin-dependent (type II) diabetic patients. Metabolism 43: 1426-1429

30. Cohen G, Heikkila RE (1974) The generation of hydrogen peroxide, superoxide radical, and hydroxyl radical by 6-hydroxydopamine, dialuric acid, and related cytotoxic agents. J Biol Chem 249: 2447-2452

31. Asayama K, Nyfeler F, English D, Pilkis SJ, Burr IM (1984) Alloxan-induced free radical production in isolated cells. Selective effect on islet cells. Diabetes 33: 1008-1011

32. Slonim AE, Surber ML, Page DL, Sharp RA, Burr IM (1983) Modification of chemically induced diabetes in rats by vitamin E. Supplementation minimizes and depletion enhances development of diabetes. J Clin Invest 71: 1282-1288

33. Ohkuwa T, Sato Y, Naoi M (1995) Hydroxyl radical formation in diabetic rats induced by streptozotocin. Life Sciences 56: $1789-1798$ 